\title{
The Treatment of Solar Lentigo using Dr. Hoon Hur's Optimal Melanocytic Suicide-2 Parameter with a High fluence 1064nm Nd:YAG Laser without Postinflammatory Hyperpigmentation
}

${ }^{*}$ Hoon Hur, Yu Ri Kim.

Choice Dermatology Clinic, Pyeongchon, Korea

Received: April 05, 2017; Accepted: April 26, 2017; Published: May 05, 2017

*Corresponding author: Hoon Hur, Choice Dermatology Clinic, 1045, Hogye-dong, Dongan-gu, Anyang-si, Gyeonggi-do, Korea, Tel: 82-31-3837533; E-mail: dermahur@naver.com

\begin{abstract}
A solar lentigo is a small, well-circumscribed, pigmented macule surrounded by normal-appearing skin. Histopathlogic findings may show epidermal hyperplasia and increased melanin pigmentation of the basal layer. A variable number of melanocytes are present; these melanocytes may be increased in number, but they do not form nests. In the most cases, treatment is not necessary for solar lentigo. However treating the solar lentigo without postinflammatory hyperpigmentation is very difficult and treatment for solar lentigo without side effects such as postinflammatory hyperpigmentation, scars and recurrences cannot be found in any literature yet. Therefore the authors introduce the new treatment of solar lentigo using optimal melanocytic suicide- 2 parameter with a high fluence $1064 \mathrm{~nm}$ Q-switched Nd:YAG laser without side effects or recurrences.
\end{abstract}

\section{Introduction}

A solar lentigo is a flat, sharply circumscribed patch. It can be round, oval or irregular in shape. Color varies from skincolored, brown to dark brown or black, and size varies from a few millimeters to several centimeters in diameter [1,2]. It results from long-term exposure to Ultraviolet (UV) radiation, which causes local proliferation of epidermal melanocytes and accumulation of melanin within the keratinocytes [3]. Solar lentigos or lentigines are very common, especially in people over the age of 40 years. But the treatment of solar lentigos or lentigines without side effects such as postinflammatory hyperpigmentation (PIH), scars and recurrences are extremely difficult [4-6]. Therefore the authors introduce the new treatment of solar lentigo using Dr. Hoon Hur's optimal melanocytic suicide-2(OMS-2) parameter with a high fluence $1064 \mathrm{~nm}$ Q-switched Nd:YAG laser without side effects or recurrences.

\section{Report of Cases}

Fifty two Korean patients with solar lentigo or solar lentigines (age range: 40-72 years old, mean age: 52.6 years) participated in this study. All patients were clinically diagnosed with solar lentigo or solar lentigines (Fig.1, 3, 5, 7, 9, 12, 15).

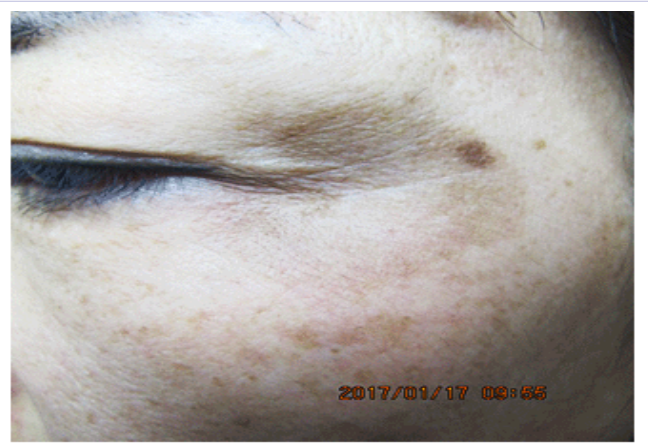

Figure 1: A single large round brown patch on the left lateral orbital rim area (Before treatment: 2017/1/17)

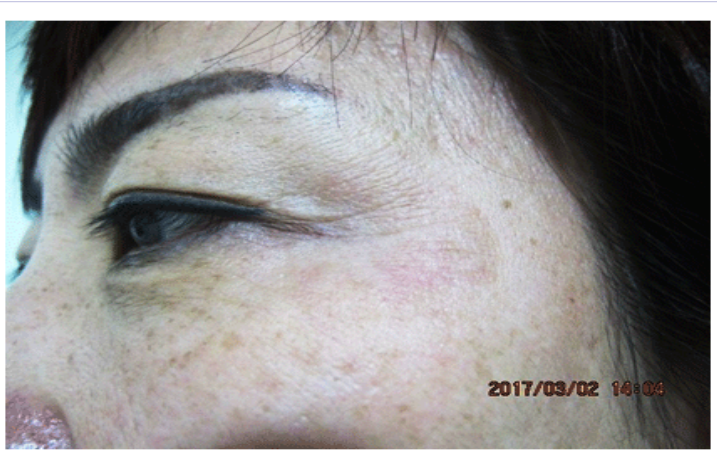

Figure 2: A complete clearance of solar lentigo

(After treatment with Dr. Hoon Hur's OMS-2 Parameter: 2017/3/2) 


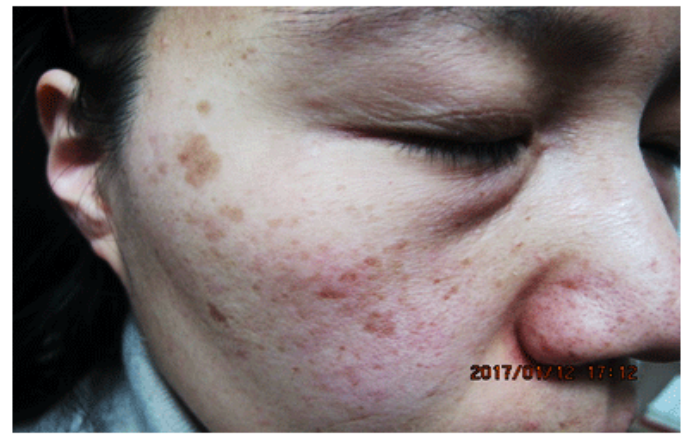

Figure 3: Multiple small well-circumscribed brown macules on the right zygoma area (Before treatment: 2017/1/12)

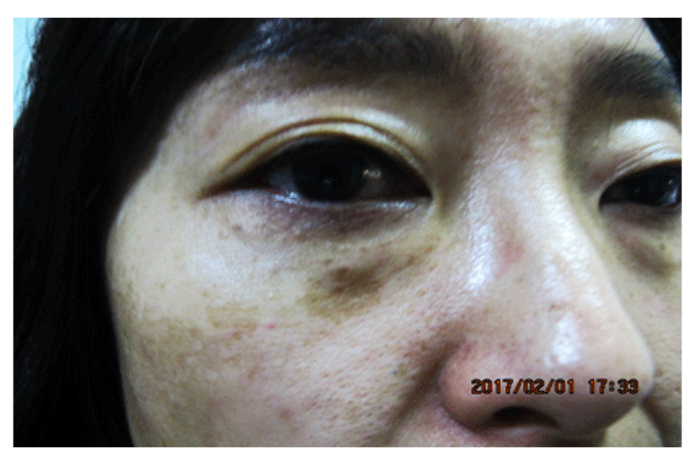

Figure 5: A single round well-circumscribed brown patch with two small round dark brown macules (Before treatment: 2017/2/1)

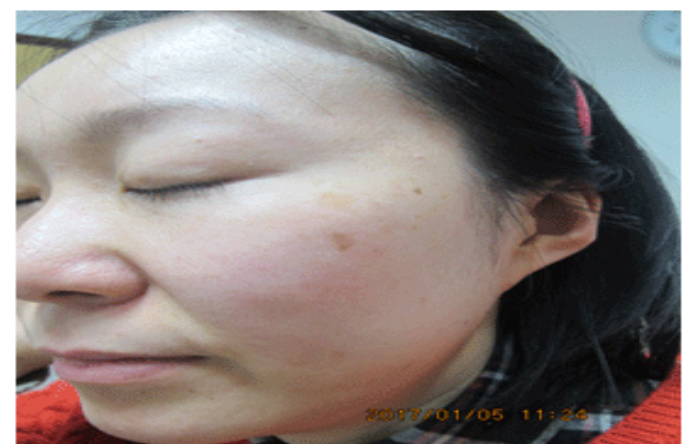

Figure 7: A Multiple small well-circumscribed brown macules on the left zygoma area (Before treatment: 2017/1/5)

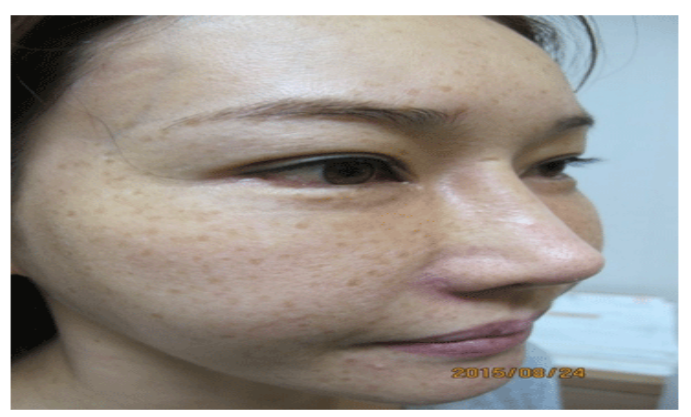

Figure 9: Multiple small round well-circumscribed brown macules on the right zygoma area and cheek (Before treatment: 2015/8/24)

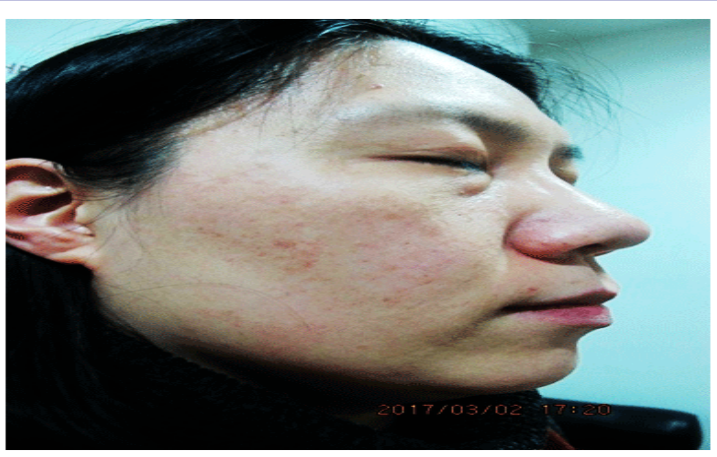

Figure 4: A complete clearance of solar lentigos

(After treatment with Dr. Hoon Hur's OMS-2 Parameter: 2017/3/2)

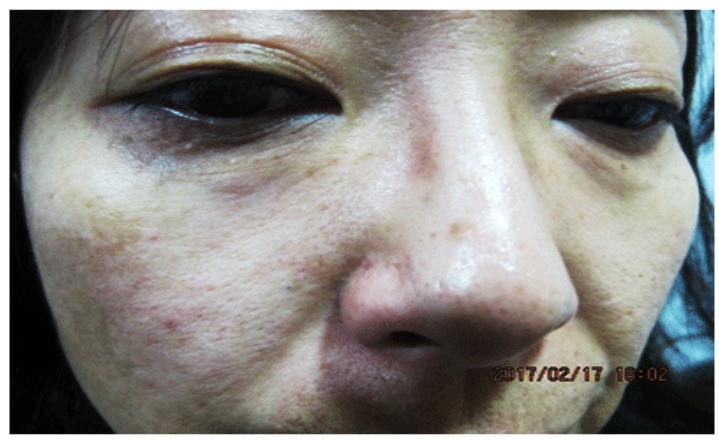

Figure 6: A complete clearance of solar lentigo (After treatment with Dr. Hoon Hur's OMS-2 Parameter: 2017/2/17)

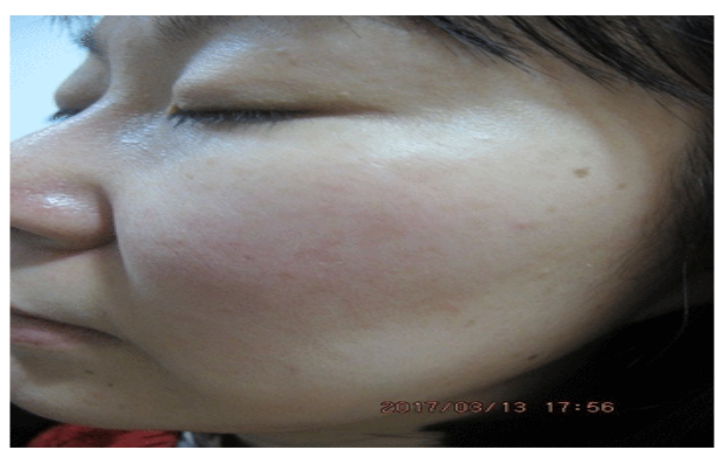

Figure 8: A complete clearance of solar lentigos

(After treatment with Dr. Hoon Hur's OMS-2 Parameter:2017/3/13)

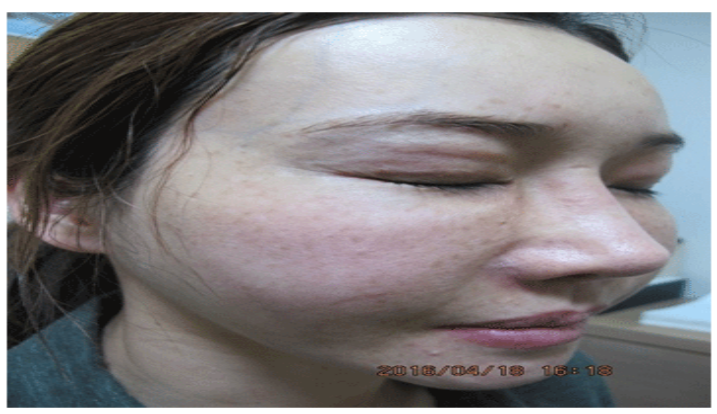

Figure 10: There is no recurrence at 6 months follow-up after the end of the treatment $(2016 / 4 / 18)$

Citation: Hoon Hur, Yu Ri Kim. (2017) The Treatment of Solar Lentigo using Dr. Hoon Hur's Optimal Melanocytic Suicide-2 Parameter with a High fluence 1064nm Nd:YAG Laser without Postinflammatory Hyperpigmentation. Clin Res Dermatol Open Access $4(2): 1-5$. 


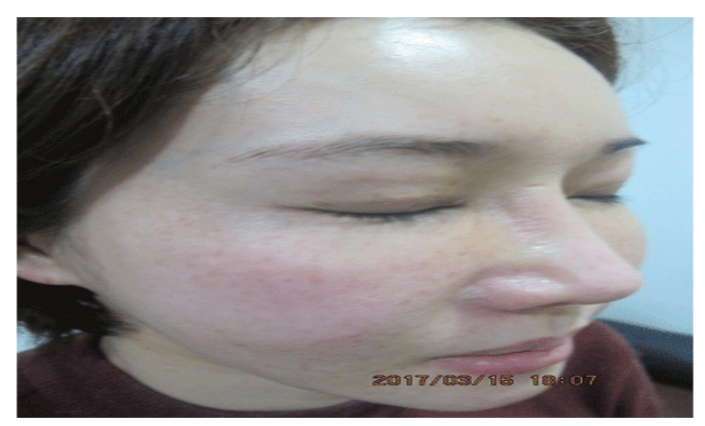

Figure 11: There is no recurrence at 18 months follow-up after the end of the treatment(2016/4/18)

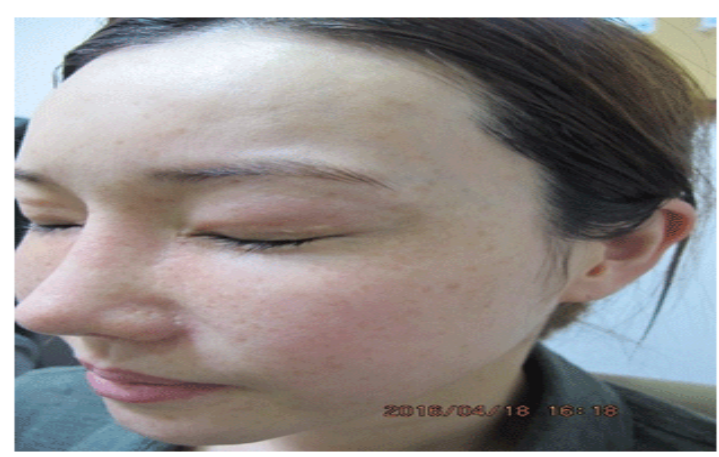

Figure 13: MA complete clearance of solar lentigos

(After treatment with Dr. Hoon Hur's OMS-2 Parameter: 2017/3/2)

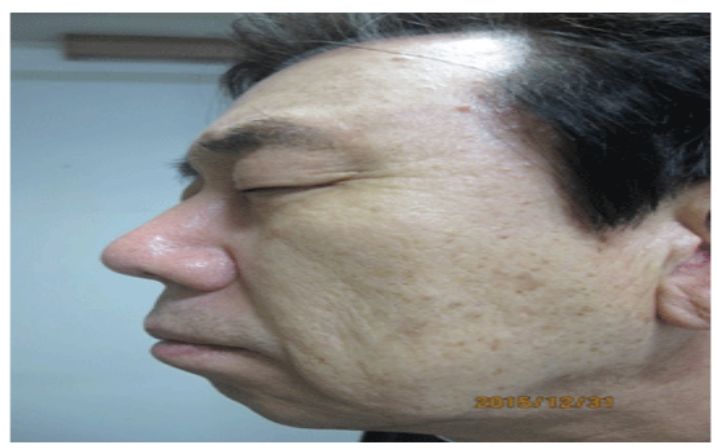

Figure 15: Multiple small round well-circumscribed brown macules on the left zygoma area and cheek (Before treatment: 2015/12/31)

Otherwise, the patients had no significant medical or familial history. After obtaining written informed consent, all of the 52 patients were subjected to 8 treatment sessions of a $1064 \mathrm{~nm}$ Q-switched Nd:YAG laser (QX-MAX Laser, Fotona, Slovenia) at a one-week interval with a spot size of $3 \mathrm{~mm}$, a fluence of $5 \mathrm{~J} / \mathrm{cm} 2$ and a pulse rate of $10 \mathrm{~Hz}$ with pulse stacking technique for 3 seconds to the solar lentigo. Ice packs were immediately applied to the entire face after laser treatment sessions, and patients were instructed to use a broad-spectrum sunscreen daily. Patient photos were obtained on the day of treatment and 4 weeks after the final session. The evaluation was performed by standardized

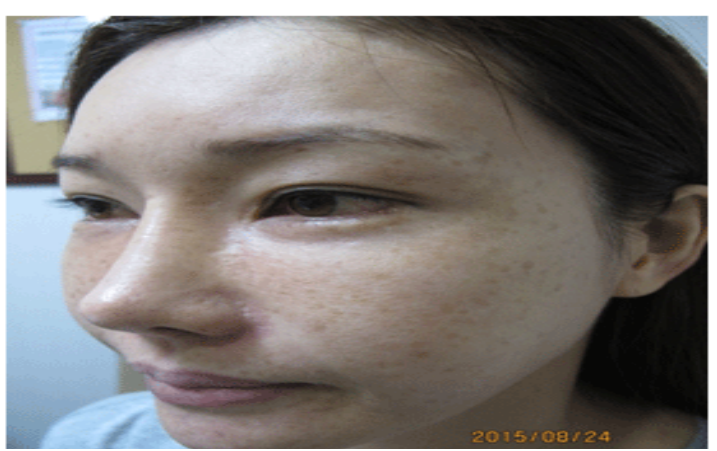

Figure 12: Multiple small round well-circumscribed brown macules on the left zygoma area and cheek (Before treatment: 2015/8/24)

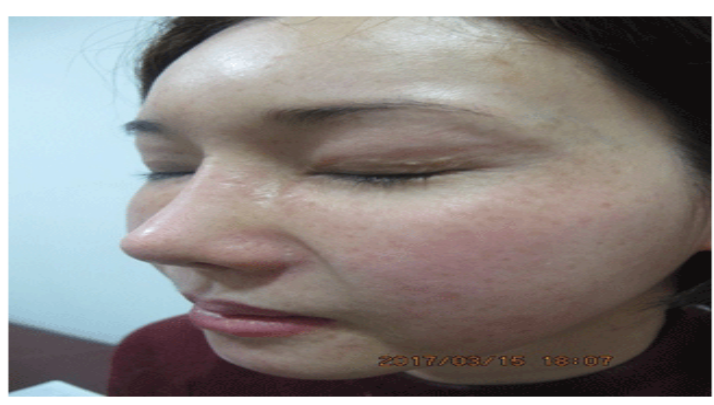

Figure 14: There is no recurrence at 18 months follow-up after the end of the treatment $(2016 / 4 / 18)$

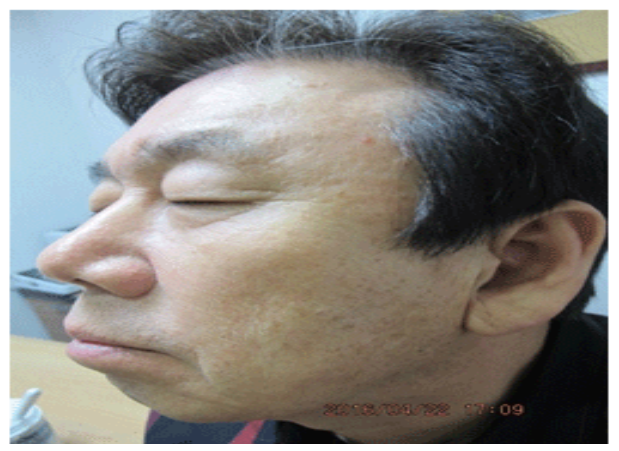

Figure 16: A complete clearance of solar lentigos (After treatment with Dr. Hoon Hur's OMS-2 Parameter: 2016/4/22)

digital photography using a Canon Camera G11 (Japan). Patients were asked to report any side effects, pain or discomfort during the treatment. All patients were satisfied with the results, and no any significant side effects, including purpura, PIH and scar except slight pain during the laser treatment (Fig. 2, 4, 6, 8, 10, $13,16)$.

\section{Discussion}

A solar lentigo (plural, solar lentigines), also known as a sun-induced freckle or senile lentigo, is a brown to dark brown lesion caused by natural or artificial ultraviolet light. Solar lentigo 
may be single or multiple [1,2]. This type of lentigo is different from a simple lentigo (lentigo simplex) because it is caused by long-term exposure to UV light. Solar lentigo is benign, but it may indicate excessive sun exposure, a risk factor for the development of skin cancer $[1,2]$. Histopathlogic findings of solar lentigo may show epidermal hyperplasia and increased melanin pigmentation in the basal layer. A variable number of melanocytes are present; these melanocytes may be increased in number, but they do not form nests [3]. In the most cases, treatment is not necessary for solar lentigo. However treating the solar lentigo without $\mathrm{PIH}$ is very difficult [4-6]. Although the precise pathogenesis of $\mathrm{PIH}$ is idiopathic, there are possible several reasons of occurrence of PIH when treating solar lentigo with conventional laser therapy. Generally $515-755 \mathrm{~nm}$ of intense pulsed light, $532 \mathrm{~nm}$ of Q- Swithched Nd:YAG laser, $694 \mathrm{~nm}$ of ruby laser and $755 \mathrm{~nm}$ of alexandrite laser are absorbed in much more melanin compared to $1064 \mathrm{~nm}$ of Q- Swithched Nd:YAG laser. Thus, the laser energy which destroys epidermal melanocytes injuries the surrounding keratinocytes, and the damaged keratinocytes secrete interleukin-1 (IL-1). IL-1 stimulates keratinocytes to secrete endothelin-1, $\alpha$-Melanocyte Stimulating Hormone (MSH), Adrenocorticotropic Hormone (ACTH) and Prostaglandin(PGE2,PGF2 $\alpha$ ). These keratinocytic injury-induced cytokines activate melanocytes and increase melanin synthesis in the melanosomes, therefore causing PIH and worsening solar lentigo [7-11]. The damged keratinocytes also secrete the single-chain urokinase type plasminogen activator (sc-uPA). The sc-uPA converts plasminogen to plasmin, which stimulates the keratinocytes to secrete basic fibroblast growth factor (bFGF). Again bFGF activates the melanocytes and increases melanin synthesis in the melanosomes, therefore causing PIH and worsening solar lentigo [7-11].

When the conventional laser therapy causes petechiae and crusts, laser energy may injury fibroblasts, mast cells, lymphocytes, macrophages, and vascular endotheliums. Then, the fibroblasts mainly secrete Stem Cell Factor (SCF) and Hepatocyte Growth Factor (HGF) which activate melanocytes and increase melanin synthesis in the melanosomes, eventually causing $\mathrm{PIH}$ and worsening solar lentigo [7-11]. Finally, reactive oxygen species such as nitric oxide, free radical oxygen and peroxide, generated from the damaged keratinocytes also activate melanocytes and increase melanin synthesis in the melanosomes, eventually causing PIH and worsening solar lentigo [7-11]. To avoid the side effects such as PIH, scarring and worsening solar lentigo of the conventional laser therapy, the authors devised Dr. Hoon Hur's optimal melanocytic suicide-2(OMS-2) parameter therapy with a high fluence $1064 \mathrm{~nm}$ Q-switched Nd:YAG laser without side effects or recurrences.

Dr. Hoon Hur's OMS-2 Parameter therapy with a high 1064nm Q-switched Nd:YAG laser may destroy epidermal melanocytes without keratinocyte damage, and the end products of damaged melanocytes will be removed through transepidermal elimination $[10,11]$. Also the end products of damaged melanocytes drop into the upper dermis. The dispersed melanosomes and melanins are removed by dermal melanophage through the lymphatic system $[10,11]$.

The authors suggest that the name of this therapy is
"Dr. Hoon Hur's OMS-2 Parameter Therapy". Because we believes that it can not only destroy epidermal melanocytes with minimal epidermal damage but also accelerate apoptotic melanocytic cell death program and can improve various skin diseases without side effects such as PIH and scarring (Table 1).

Table1: Indication of Dr. Hoon Hur's OMS-2 Parameter therapy

Solar lentigo or Solar lentigines

Multiple lentigines or facial lentiginosis

Macular seborrheic keratosis

Acquired bilateral nevus of Ota-like macules(ABNOM)

PIH

Melanocytic nevus

Verruca plana

The authors think that the mechanism of Dr. Hoon Hur's OMS-2 Parameter Therapy is to destroy the epidermal melanocytes and minimize the epidermal damage without petechiae and crusts because a wavelength of $1064 \mathrm{~nm}$ result in less absorption by epidermal melanin. Then, weekly OMS-2 Parameter Therapy destroys melanocytes completely and accelerates apoptotic melanocyte cell death, thus the end products of damaged melanocytes such as the dispersed melanosomes and melanins are either removed by the transepidermal elimination or are removed by dermal melanophages through the lymphatic system $[10,11]$. Eventually, complete clearance of solar lentigo without side effects and recurrences can be achieved. In these cases, our patients with solar lentigo were treated with 8 sessions of a high fluence1064nm Q-switched Nd:YAG laser (QX-MAX Laser, Fotona, Slovenia) at a one-week interval with a spot size of $3 \mathrm{~mm}$, a fluence of $5 \mathrm{~J} / \mathrm{cm}^{2}$ and a pulse rate of $10 \mathrm{~Hz}$ with pulse stacking technique for 3 seconds to the solar lentigo. The pulse stacking technique for 3 seconds to the solar lentigo is very important to destroy the epidermal melanocytes with minimizing the epidermal damage. If the pulse stacking technique for more than 3 seconds to the solar lentigo was performed, the epidermal damages might have occurred and the damaged keratinocytes might have secreted the keratinocytic injury-induced cytokines such as endothelin-1, $\alpha$-MSH, ACTH, bFGF, and prostaglandin (PGE2, PGF2 $\alpha$ ) which activate melanocytes and increase melanin synthesis in the melanosomes, therefore causing PIH and worsening solar lentigo [7-11]. In case of solar lentigo on face, 8 sessions of OMS-2 Parameter Therapy are performed once a week. But in case of solar lentigo on other parts of body (arms, legs, and torso), more than 8 sessions of OMS-2 Parameter Therapy should be performed once a week regardless of the lesional size. The merit of OMS-2 Parameter Therapy is that it minimizes the epidermal damage without petachiae and crusts. This parameter can deliver the sufficient energy to destroy epidermal melanocytes and also salvage normal background tissue due to less absorption by epidermal mela- 
nin. Therefore this therapy does not provoke PIH and scarring But OMS-2 Parameter Therapy requires 8 treatment sessions for 8 weeks. In these cases, 52 patients with a solar lentigo or solar lentigines (Fig. 1,3,5,7,9,12,15) were treated with OMS-2 Parameter of a high fluence $1064 \mathrm{~nm}$ Q-switched Nd:YAG laser. A total of 52 patients with a solar lentigo or solar lentigines was achieved complete clearance of the pigmented lesions without PIH (Fig. 2, $4,6,8,10,13,16)$. There are no recurrences at 18 months followup (Fig.11, 14). All patients were satisfied with the results of Dr. Hoon Hur's OMS-2 Parameter Therapy, and no any significant side effects, including PIH and scarring .

\section{Conclusion}

The parameter for each Dr. Hoon Hur's OMS-2 Parameter Therapy was a spot size of $3 \mathrm{~mm}$, a fluence of $5 \mathrm{~J} / \mathrm{cm} 2$ and a pulse rate of $10 \mathrm{~Hz}$ with pulse stacking technique for 3 seconds. This therapy does not cause side effects such as petechiae, crusts and PIH during the laser treatment. There is no recurrence at 18 months follow-up. Therefore, Dr. Hoon Hur's OMS-2 Parameter Therapy is thought that more effective and safe results of treatment than conventional laser treatment can be expected.

\section{References}

1. Derancourt C, Bourdon-Lanoy E, Grob JJ, Guillaume JC, Bernard P, Bastuji-Garin S. Multiple large solar lentigos on the upper back as clinical markers of past severe sunburn: a case-control study. dermatology 2007;214(1):25-31.

2. Byrom L, Barksdale S, Weedon D, Muir J. Unstable solar lentigo: A defined separate entity. Australas J Dermatol. 2016;57:229-234. doi: 10.1111/ajd.12447

3. Andersen WK, Labadie RR, Bhawan J. Histopathology of solar lentigines of the face: a quantitative study. J Am Acad Dermatol. 1997;36(3 Pt 1):444-447.
4. Rosenbach A, Lee SJ, Johr RH. Treatment of medium brown solar lentigines using an Alexandrite laser designed for hair reduction. Arch Dermatol 2002;138(4):547-548.

5. Rosenback A, William CW, Alster TS. Comparison of the Q-Switched Alexandrite (755nm) and Q-Switched Nd:YAG (1064nm) lasers in the treatment of benign melanocytic nevi. Dermatol Surg. 1997;23:239244.

6. Traeli J, Kwan JM, Meehan KJ, Domankivitz Y, Gilbert S, Malomo K, et al. Use of a long-pulsed Alexandrite laser in the treatment of superficial pigmented lesions. Dermatol Surg. 2007;33(12):1-7.

7. Hattori H, Kawashima M, Ichikawa Y, Imokawa G. The epidermal stem cell factor is over-expressed in lentigo senilis: Implication for the mechanism of hyper pigmentation. J Invest Dermatol. 2004;122(5):12561265.

8. Okazaki M, Yoshimura K, Suzuki Y, Uchida G, Kitano Y, Harii K, et al. The mechanism of epidermal hyperpigmentation in cafe au lait macules of neurofibromatosis type 1 may be associated with dermal fibroblastderived stem cell factor and hepatocyte growth factor. Bri J Dermatol. 2003;148(4):689-697.

9. Okazaki M, Youshimura K, Uchida G, Suzuki Y, Kitano Y, Harii K. Epidermal hyperpigmentation in non-syndromic solitary cafe au lait macules may be associated with increased secreation of endothelin- 1 by lesional keratinocytes. J Plastic Sur \& hand Sur. 2005;39(4):213-217.

10. Hur H. The treatment of café au lait spot using Dr.Hoon Hur's Golden Parameter Therapy. J Dermatol Ther. 2016;1(1):1-4

11. Hur H, Kim YR, Shim DT. The treatment of cafe au lait spot, partial unilateral lentiginosis and Becker's nevus using a high fluence 1064nm Q-swithed Nd: YAG laser. J Clin and Cosmet Dermatol. 2017;1:1-4. 